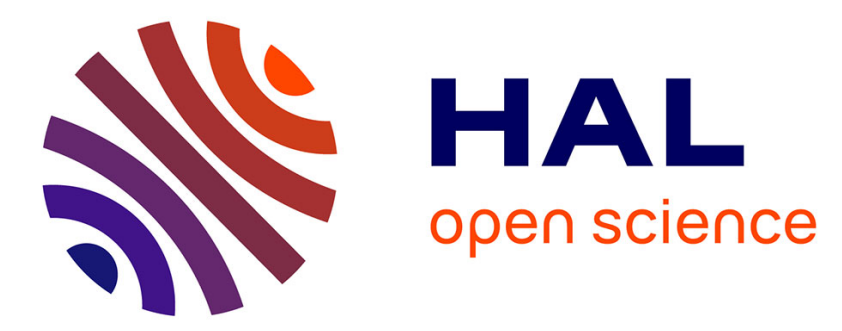

\title{
FDMX: extended X-ray absorption fine structure calculations using the finite difference method
} Jay D. Bourke, Christopher T. Chantler, Yves Joly

\section{To cite this version:}

Jay D. Bourke, Christopher T. Chantler, Yves Joly. FDMX: extended X-ray absorption fine structure calculations using the finite difference method. Journal of Synchrotron Radiation, 2016, 23, pp.551559. 10.1107/S1600577516001193 . hal-01279479

\section{HAL Id: hal-01279479 \\ https://hal.science/hal-01279479}

Submitted on 26 Feb 2016

HAL is a multi-disciplinary open access archive for the deposit and dissemination of scientific research documents, whether they are published or not. The documents may come from teaching and research institutions in France or abroad, or from public or private research centers.
L'archive ouverte pluridisciplinaire $\mathbf{H A L}$, est destinée au dépôt et à la diffusion de documents scientifiques de niveau recherche, publiés ou non, émanant des établissements d'enseignement et de recherche français ou étrangers, des laboratoires publics ou privés. 


\title{
FDMX: extended X-ray absorption fine structure calculations using the finite difference method
}

\author{
Jay D. Bourke, Christopher T. Chantler and Yves Joly
}

J. Synchrotron Rad. (2016). 23, 551-559

\section{IUCr Journals CRYSTALLOGRAPHY JOURNALS ONLINE}

Copyright (C) International Union of Crystallography

Author(s) of this paper may load this reprint on their own web site or institutional repository provided that this cover page is retained. Republication of this article or its storage in electronic databases other than as specified above is not permitted without prior permission in writing from the IUCr.

For further information see http://journals.iucr.org/services/authorrights.html 
JOURNAL OF SYNCHROTRON RADIATION

ISSN 1600-5775

Received 13 November 2015

Accepted 19 January 2016

Edited by R. W. Strange, University of Liverpool, UK

Keywords: X-ray absorption; finite difference methods; fine structure; XAFS; FDMNES; FDMX.

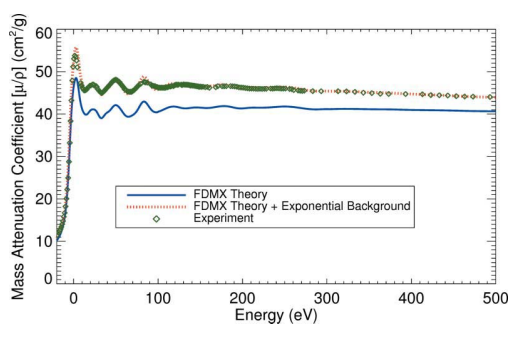

(C) 2016 International Union of Crystallography

\section{FDMX: extended X-ray absorption fine structure calculations using the finite difference method}

\author{
Jay D. Bourke, ${ }^{a}$ Christopher T. Chantler ${ }^{a *}$ and Yves Joly ${ }^{b, c}$ \\ aSchool of Physics, University of Melbourne, Australia, 'b Université Grenoble Alpes, Institut NÉEL,

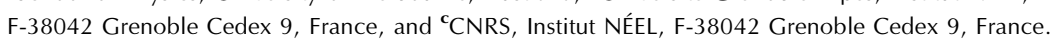 \\ *Correspondence e-mail: chantler@unimelb.edu.au
}

A new theoretical approach and computational package, $F D M X$, for general calculations of X-ray absorption fine structure (XAFS) over an extended energy range within a full-potential model is presented. The final-state photoelectron wavefunction is calculated over an energy-dependent spatial mesh, allowing for a complete representation of all scattering paths. The electronic potentials and corresponding wavefunctions are subject to constraints based on physicality and self-consistency, allowing for accurate absorption cross sections in the near-edge region, while higher-energy results are enabled by the implementation of effective Debye-Waller damping and new implementations of second-order lifetime broadening. These include inelastic photoelectron scattering and, for the first time, plasmon excitation coupling. This is the first full-potential package available that can calculate accurate XAFS spectra across a complete energy range within a single framework and without fitted parameters. Example spectra are provided for elemental $\mathrm{Sn}$, rutile $\mathrm{TiO}_{2}$ and the $\mathrm{FeO}_{6}$ octahedron.

\section{Introduction}

$\mathrm{X}$-ray absorption fine structure (XAFS) refers to the oscillations in the energy-dependent photo-absorption coefficient of a condensed matter system, commonly seen at energies up to a few hundred $\mathrm{eV}$ above an ionization edge. These oscillations are the result of self-interference of photoelectron excitations, which have scattered elastically from one or more local atoms in the material. The precise form of an XAFS spectrum is directly determined by the spatially dependent elastic and inelastic electron scattering coefficients of the material, and therefore is a function of the complex electronic potential within a critical region near the ionized atom.

The most obvious determinant of this potential is the position of neighbouring atoms, and particularly their associated electron densities. This makes XAFS an excellent probe of local molecular structure, not only for simple elements and solids but also for large compounds, aqueous samples, gases and amorphous materials. The physical structure around a central atom can be probed by tuning the X-ray energy to be near an inner-shell ionization energy for a specific element, and in this way co-ordination numbers, crystal groups and bond lengths can be measured routinely for materials which do not lend themselves to effective study with other crystallographic methods.

The relationship between XAFS and the local complex potential has many other significant consequences. At energies very close to the absorption edge (i.e. photoelectron energies less than $60 \mathrm{eV}$ ), the XAFS spectrum is highly sensitive to minor changes in the potential that may be associated with changes to ionization state, oxidation state and 
bonding effects. This XANES region is also very sensitive to artificial structures that commonly occur in many theoretical XAFS models, meaning that to accurately quantify XANES spectra a full-potential model such as the finite difference method (FDM) or linearized augmented plane-wave (LAPW) method is required.

Such methods have seen long-standing use in XANES calculations, but have been less popular for XAFS analysis than multiple-scattering approaches, which utilize approximated and effective potentials in order to consider photoelectron scattering on a path-dependent basis. Multiplescattering approaches are substantially more efficient than full-potential models, and have advantages with respect to fitting and analysis, as the contribution to the spectrum of individual scattering paths can be readily quantified. Therefore, explicitly path-dependent effects such as thermal motion and, to a lesser extent, inelastic scattering could be modelled more robustly. These issues tend to be more significant for higher photoelectron energies, and have meant that fullpotential modelling has mostly been restricted to XANES spectra.

Recent advances, however, have demonstrated that the FDM can be utilized for high-energy calculations using appropriate implementation of thermal and lifetime broadening effects, valence-shell contributions, and a sufficiently high-precision representation of the electronic and exchangecorrelation potentials within a small spherical cluster (Chantler \& Bourke, 2014a; Bourke \& Chantler, 2010a). This has enabled the development of a new package, presented here, capable of robust computation of X-ray absorption spectra ranging from below an absorption edge up to photoelectron energies of several $\mathrm{keV}$, corresponding to the high-energy atom-like photo-absorption limit.

\section{The Finite Difference Method for XAFS}

The FDM is a common mathematical procedure for evaluating the solution to differential equations by approximating local derivatives over a discretized grid. At each grid point, these derivatives are linked together via a series of linear equations, enabling the determination of function values at any point provided appropriate boundary conditions are chosen. With respect to XANES or XAFS calculations, this technique is employed to solve the Schrödinger equation, facilitating the determination of electron wavefunctions and subsequently the transition matrix elements for photoelectric ionization.

The first application of the FDM to this problem was in the Finite Difference Method for Near-Edge Structure (FDMNES) package (Joly, 2001). This package has become one of the foremost computational tools for XANES analysis due to its physical representation of electronic potentials for low-energy spectra, and robust applicability to finite molecular structures.

Here we present the Finite Difference Method for XAFS $(F D M X)$ package, a significant enhancement built upon the original FDMNES in order to calculate extended XAFS spectra in a similarly robust and physical manner. As in FDMNES, FDMX calculations treat the problem of XAFS in a relatively general way, starting from the basic need to determine the optical transition matrix elements which, in the quadrupolar approximation, may be written as

$$
M_{g f}=\left\langle\psi_{f}|\boldsymbol{\varepsilon} \cdot \mathbf{r}[1+(i / 2) \mathbf{k} \cdot \mathbf{r}]| \psi_{g}\right\rangle,
$$

where $\mathbf{k}$ is the photon wavevector polarized in the $\boldsymbol{\varepsilon}$ direction, and $\psi_{g}$ and $\psi_{f}$ are the initial and final states for the absorbing electron. The transition amplitudes are then summed to give the absorption cross section $\sigma$ following

$$
\sigma=4 \pi^{2} \alpha \hbar \omega \sum_{f, g}\left|M_{g f}\right|^{2} \delta\left(\hbar \omega-E_{f}+E_{g}\right),
$$

where $\alpha$ is the fine-structure constant, $\hbar \omega$ the energy of the incident photon, and $E_{f}, E_{g}$ the final and initial state energies. The initial state is that of an electron bound to an atomic core orbital, and is approximated via a relativistic Dirac-Slater model (Rosen \& Ellis, 1975). The final state is calculated from the cluster potential using the FDM.

The FDM solves a large number of simultaneous linear equations linking the values of the wavefunctions $\psi_{i}$, and potentials $V_{i}$, at points $i$ in a defined grid in real space. The Laplacian operator needed to solve the Schrödinger equation is approximated using a fourth-order polynomial and, in the case of a cubic grid, may be written as

$$
\nabla^{2} \psi_{i}=\frac{1}{h^{2}}\left(\frac{4}{3} \sum_{j, \varepsilon} \psi_{j}^{\varepsilon}-\frac{1}{12} \sum_{j, \varepsilon} \psi_{j}^{\varepsilon \varepsilon}-\frac{15}{2} \psi_{i}\right) .
$$

Here $\varepsilon=+$ or,$- \psi_{j}^{\varepsilon}$ and $\psi_{j}^{\varepsilon \varepsilon}$ are the first and second nearest neighbouring grid points to $i$ in the direction $\varepsilon j$, and $h$ is the distance between neighbouring grid points. A hexagonal mesh is also used to more efficiently model crystal groups with corresponding hexagonal symmetry. In that case, there are six first and six second neighbour points (instead of four) in the plane perpendicular to the three-fold axis. The corresponding Laplacian operators are then multiplied by $2 / 3$ compared with the cubic mesh. Of course, the operators for the neighbour points along the three-fold axis are not modified. With our approximated Laplacian, we write the non-relativistic Schrödinger equation for a non-magnetic material in discretized form as

$$
\left(-\nabla_{i i}^{2}+V_{i}-E\right) \psi_{i}+\sum_{j}\left(-\nabla_{i j}^{2} \psi_{j}\right)=0
$$

where again $\psi_{j}$ refers to wavefunction values at grid points neighbouring $i$. The values of the wavefunction are initially determined for all points $i$ following a first-order estimate of the potentials $V_{i}$. These are approximated by the Coulomb potential induced by the ground-state electron densities plus an exchange-correlation potential, by default approximated using the Hedin-Lundqvist theory (Hedin \& Lundqvist, 1971; Joly et al., 1999). Electron densities are initially approximated via an atomistic Dirac-Hartree-Fock algorithm, which is highly accurate for the majority of bound electrons contributing to the XAFS spectrum, particularly including the core of the absorbing atom. The wavefunctions and potentials are then iterated in order to converge to a self-consistent result 
within the discretized regions of the spherical cluster. In this fashion the total electron number within the cluster is constrained to match the sum of ground-state contributions from each atom.

The code also provides the possibility of relativistic calculations which increase the complexity of the equations slightly with a spin-orbit term depending on the gradient of the potential and doubling the basis with spin up and spin down wavefunction components. What is presented here therefore applies also to relativistic and magnetic materials.

The FDM algorithm is applied only in an interstitial region between atoms. In regions close to atomic cores, the shape of the potential demands prohibitively high grid densities, and so instead a model is used in which the potential is approximated as symmetric, and the wavefunction is evaluated as a sum of spherical harmonics. These wavefunctions are then matched via continuity conditions to the values of the wavefunction at the edge of the atomic cores, which are typically around $0.6 \AA$ in radius. The code also optionally offers the possibility of using a non-spherical potential inside this sphere, giving a set of expansion of radial wavefunctions depending on both $l$ and $m$ quantum numbers. So far we have not found cases where this was necessary. In a similar manner, wavefunctions outside of the spherical cluster (usually centred at the absorbing atom) comprise Neumann and Bessel functions.

The radius of the spherical cluster used for the calculation is chosen such that contributions from atoms lying a greater distance from the absorber would be expected to be negligible. A typical choice for the cluster radius may be around 6-8 $\AA$ at low photoelectron energies, but for extended XAFS these radii may be reduced due to the effects of inelastic scattering and thermal motion.

It is useful to define $h$ as the distance between neighbouring grid points, the value of which ultimately determines the precision of the representation of the potential. For XANES calculations and FDMNES, it is usually sufficient to use a constant value for $h$ of around $0.25 \AA$. In $F D M X$, however, $h$ is reduced as a function of photoelectron energy in order to maintain convergence of the wavefunction across the entire XAFS spectrum.

\section{Thermal effects}

FDMX implements thermal effects via a Debye-Waller formalism based on the correlated Debye model of Beni \& Platzman (1976). For explicitly path-dependent theories, it is common to parameterize thermal motion via an array of pathdependent effective isotropic thermal parameters (ITPs) $\sigma_{j}^{2}$, corresponding to the mean-square relative displacements between the photo-absorbing atom and its nearby neighbours. Higher-order ITPs may also be used for multiple-scattering paths when the scattering atoms are not arranged co-linearly (Rehr et al., 2009). However, it is not currently practicable for any model to treat these parameters independently, and so an effective ITP $\sigma_{\text {eff }}^{2}$ is used, which may be based on the dominant scattering path. For most structures, this approximates to the shortest or most degenerate scattering path. The data can certainly be sensitive to multiple ITPs from dominant paths.

The ITP for any given scattering path is given in terms of the mean square relative displacements of the absorbing atom, $\left\langle\left(u_{0} R_{j}\right)^{2}\right\rangle$, its neighbour atom $\left\langle\left(u_{j} R_{j}\right)^{2}\right\rangle$ and the displacement correlation function $\left\langle\left(u_{0} R_{j}\right)^{2}\left(u_{j} R_{j}\right)^{2}\right\rangle$, following

$$
\sigma_{j}^{2}=\left\langle\left(u_{0} R_{j}\right)^{2}\right\rangle+\left\langle\left(u_{j} R_{j}\right)^{2}\right\rangle-2\left\langle\left(u_{0} R_{j}\right)^{2}\left(u_{j} R_{j}\right)^{2}\right\rangle .
$$

$u_{0}$ is the instantaneous displacement of the photo-absorbing atom from its equilibrium position, $u_{j}$ is the same for some neighbouring atom $j$, and $R_{j}$ is a unit vector pointing from the photo-absorber to atom $j$. The displacement of atoms within the absorbing material arises due to the propagation of resonant phonons of energy $\hbar \omega_{q \lambda}$, where $\hbar q$ is the phonon momentum and $\lambda$ is the polarization index. In the special case of a monoatomic crystal, one can write the mean square relative thermal displacement in terms of these phonon resonances following (Beni \& Platzman, 1976)

$$
\sigma_{j}^{2}=\frac{\hbar}{m} \sum_{q \lambda}\left(\varepsilon_{q \lambda} R_{j}\right)^{2} \frac{1}{\omega_{q \lambda}} \operatorname{coth}\left(\frac{\hbar \omega_{q \lambda}}{2 k_{\mathrm{B}} T}\right)\left[1-\cos \left(q R_{j}\right)\right],
$$

where $m$ is the atom mass, $k_{\mathrm{B}}$ the Boltzmann constant, $\varepsilon_{q \lambda}$ is a unit vector in the direction of polarization, and $T$ is the temperature of the material. A value for $T$ may be provided by the user of $F D M X$, or will otherwise default to $298 \mathrm{~K}$. In general, determination of the phonon spectrum $\omega_{q \lambda}$ can be performed explicitly via experiment (Fornasini et al., 2004) or theory (Vila et al., 2007), or may be estimated using an Einstein, Debye or more complex model. In $F D M X$, a Debye model is used, which leads to the following expression for the XAFS ITP (Greegor \& Lytle, 1979),

$$
\begin{aligned}
\sigma_{j}^{2}= & \frac{6 \hbar}{m \omega_{\mathrm{D}}}\left[\frac{1}{4}+\left(\frac{T}{\theta_{\mathrm{D}}}\right)^{2} D_{1}\right]-\frac{6 \hbar}{m \omega_{\mathrm{D}}}\left\{\frac{1-\cos \left(q_{\mathrm{D}} R_{j}\right)}{2\left(q_{\mathrm{D}} R_{j}\right)^{2}}+\left(\frac{T}{\theta_{\mathrm{D}}}\right)^{2}\right. \\
& \left.\times\left[D_{1}-\frac{1}{3 !}\left(q_{\mathrm{D}} R_{j} \frac{T}{\theta_{\mathrm{D}}}\right)^{2} D_{3}+\frac{1}{5 !}\left(q_{\mathrm{D}} R_{j} \frac{T}{\theta_{\mathrm{D}}}\right)^{4} D_{5}-\ldots\right]\right\} .
\end{aligned}
$$

$\theta_{\mathrm{D}}, \omega_{\mathrm{D}}$ and $q_{\mathrm{D}}$ are, respectively, the Debye temperature, frequency and wavenumber. These parameters are related by

$$
\begin{gathered}
\omega_{\mathrm{D}}=\theta_{\mathrm{D}} k_{\mathrm{B}} / \hbar, \\
k_{\mathrm{D}}=\left(6 \pi^{2} / V\right)^{1 / 3},
\end{gathered}
$$

where $V$ is the mean volume per atom in the material. The $D_{n}$ parameters are definite integrals given by

$$
D_{n}=\int_{0}^{\theta_{\mathrm{D}} / T} \frac{x^{n}}{\exp (x)-1} \mathrm{~d} x .
$$

This formalism enables a sensible estimate of the ITP $\sigma_{j}^{2}$ of the dominant scattering path $j$, and hence a sufficiently accurate value for the effective ITP $\sigma_{\text {eff }}^{2}$ in many applications. In the high-energy limit, for a mono-atomic cubic structure, it has been shown to approach the correct experimental value from 
crystallography (Tantau et al., 2015). The ITP is implemented within $F D M X$ by a direct adjustment to the calculated absorption cross section following

$$
\begin{aligned}
\mu(k) \rightarrow & \mu(k) \exp \left(-2 \sigma_{\mathrm{eff}}^{2} k^{2}\right) \\
& +\mu_{0}(k)\left[1-\exp \left(-2 \sigma_{\mathrm{eff}}^{2} k^{2}\right)\right],
\end{aligned}
$$

where, from standard XAFS notation, $k$ is the photoelectron wavenumber, $\mu(k)$ is the total cross section, and $\mu_{0}(k)$ is the associated atom-like background contribution from the ionized shell, discussed in $§ 5$. For near-edge studies and complex molecular structures, $\sigma_{\text {eff }}^{2}$ typically provides a good estimate for the thermal contributions despite approximations in the derivation of its value. Errors in the final XAFS spectrum arising from these approximations tend to be quite small. In particular, at high photoelectron energies the impact of inelastic electronic scattering processes tends to be more significant, while errors from the thermal contribution in the XANES region are typically much smaller than those that are introduced by the use of an incomplete or heavily approximated electronic potential (Glover et al., 2007).

Nevertheless, it is possible, and may often be appropriate, for the user to provide their own values for $\sigma_{\text {eff }}^{2}$ or for $\theta_{\mathrm{D}}$ for particular cases, as is common with other theory packages. This can be done using the DWfactor or TDebye keywords. Such extra inputs are particularly recommended for lowtemperature cases where zero-point motion may be significant, or for samples with high static disorder, which similarly may be modelled via an additive contribution to the effective ITP.

\section{Inelastic photoelectron scattering}

While it is the elastic (coherent) scattering of the excited photoelectron that is responsible for XAFS spectra, it is the presence of inelastic scattering that imparts its most useful property, a preferential measurement of the electronic and physical structure in a localized region near the absorbing atom (Bourke \& Chantler, 2010b). The rate of inelastic scattering determines the effective size of the region being probed by XAFS, and in this application is most conveniently quantified in terms of the electron inelastic mean free path (IMFP). The IMFP is formally defined as the mean distance travelled by an electron of a given energy between successive inelastic collisions (Powell \& Jablonski, 2009).

Much progress has been made in recent years in the development of theoretical determinations for the IMFP, particularly in terms of optical data models (Sorini et al., 2006; Denton et al., 2008; Bourke \& Chantler, 2012; Da et al., 2014). These models utilize various algorithms to generalize known optical scattering data into electron scattering data, typically within statistical and independent oscillator approximations (Tung et al., 1979), and originally gained popular use with the advent of the Penn algorithm (Penn, 1987). FDMX implements a new approach, but follows a more general formalism including coupling between electron excitation channels. The approach, known as the coupled-plasmon model, is presented in full elsewhere (Bourke \& Chantler, 2015), and briefly summarized here.

Within the first Born approximation, the cross section for stimulated electronic transitions is proportional to the imaginary part of the negative inverse of the dielectric constant of the material $\varepsilon(q, \omega)=\varepsilon_{1}+i \varepsilon_{2}$ (Nikjoo et al., 2012), commonly known as the energy loss function (ELF), i.e. ELF = $\operatorname{Im}[-1 / \varepsilon(q, \omega)]=\varepsilon_{2} /\left(\varepsilon_{1}^{2}+\varepsilon_{2}^{2}\right)$. As such, the probability of an energetic electron undergoing a scattering event and depositing energy $\hbar \omega$ and momentum $\hbar q$ into a material is proportional to the integral of this loss function over all applicable energy and momenta for that electron. We therefore express the electron IMFP, $\lambda(E)$, as (Tanuma et al., 1991)

$$
\lambda^{-1}(E)=\frac{\hbar}{a_{0} \pi E} \int_{0}^{\left(E-E_{\mathrm{F}}\right) / \hbar} \int_{q_{-}}^{q_{+}} \frac{1}{q} \operatorname{Im}\left[\frac{-1}{\varepsilon(q, \omega)}\right] \mathrm{d} q \mathrm{~d} \omega,
$$

where $E$ is the electron energy, $E_{\mathrm{F}}$ the Fermi energy, $a_{0}$ the Bohr radius, and the $q \pm$ momentum limits are determined kinematically. Therefore it is only necessary to determine the electron ELF for the material, which can be done via a generalization of the optical ELF, $\operatorname{Im}[-1 / \varepsilon(0, \omega)]$, corresponding to the low-momentum limit. This definition is due to the relative insignificance of the photon momentum compared with a propagating electron, and allows us to generalize equation (12) in terms of some externally determined optical ELF (Bourke \& Chantler, 2015),

$$
\begin{aligned}
\lambda(E)_{N}^{-1}= & \frac{\hbar}{a_{o} \pi E} \int_{0}^{\left(E-E_{\mathrm{F}}\right) / \hbar} \int_{q_{-}}^{q_{+}} \int_{0}^{\infty} \frac{2}{\pi} \frac{\omega^{\prime}}{q} \\
& \times \operatorname{Im}\left[\frac{-1}{\varepsilon_{\mathrm{data}}\left(0, \omega^{\prime}\right)}\right] \\
& \times \operatorname{Im}\left[\frac{-1}{\varepsilon_{\mathrm{M}}\left(q, \omega, \gamma_{i}(q)_{N-1} ; \omega_{p}=\omega_{i}\right)}\right] \mathrm{d} \omega^{\prime} \mathrm{d} q \mathrm{~d} \omega \\
& \gamma_{i}(q)_{N}=\left.\hbar \frac{\mathrm{d} \omega_{q}}{\mathrm{~d} q}\right|_{\omega_{q}, q} \lambda(E)_{N}^{-1} \Theta(N-\delta) \\
& q \pm=\left(\frac{q^{2}}{\hbar \omega} E\right)^{1 / 2} \pm\left[\frac{q^{2}}{\hbar \omega}(E-\hbar \omega)\right]^{1 / 2}
\end{aligned}
$$

The dielectric function $\varepsilon_{\mathrm{M}}$ is a Mermin function (Mermin, 1970), and this expression can uniquely describe the evolution of electronic excitation channels with increasing momentum. It includes a broadening term $\gamma_{i}(q)$, the inverse of the lifetime of excitations, and in this formalism is determined selfconsistently in terms of the electron IMFP and excitation group velocity $\mathrm{d} \omega_{q} / \mathrm{d} q$. $\Theta$ is a Heaviside step function, $\delta$ a positive infinitesimal, and $N$ is an iteration index, which typically enables the formula to achieve convergence when $N$ is set to a value higher than 3 .

This approach is robust, self-consistent and produces IMFP values in better agreement with experiment than other techniques (Bourke \& Chantler, 2015), but requires an input of $\operatorname{Im}\left[-1 / \varepsilon_{\text {data }}(0, \omega)\right]$, corresponding to the optical loss function 
of the material. This spectrum can be determined experimentally, or via density functional packages such as WIEN2k (Blaha et al., 2001; Ambrosch-Draxl \& Sofo, 2006). For highly accurate analysis of XANES spectra, or for complex molecular structures, it is recommended that $F D M X$ users provide values for this function where possible, using the ELFin input keyword. This enables a fully self-consistent implementation of inelastic scattering effects, including plasmon coupling effects, not currently available in other packages.

Users can also provide IMFP data via the IMFPin keyword. In the absence of optical ELF or IMFP data, FDMX will utilize tabulated data from Tanuma et al. (2011) where available, which approximately corresponds to a first-order implementation of equation (13) (i.e. with $N=1$ ). If data are not available, an IMFP estimate will be made according to the TPP-2M equation (Tanuma et al., 2011). These latter estimates are less robust in the XANES region, below around $60 \mathrm{eV}$, however as with the thermal processes tend to contribute less error than the use of an incomplete or approximated potential, as is common with other XAFS packages.

\section{Lifetime broadening and background absorption}

The inelastic photoelectron scattering contributes a broadening function $\Gamma_{\lambda}(E)$ to the XAFS spectrum, corresponding to the photoelectron lifetime $\tau_{\lambda}(E)=1 / \Gamma_{\lambda}(E)$. It is therefore simply related to the electron IMFP following

$$
\Gamma_{\lambda}(E)=\frac{\hbar}{\lambda(E)}\left(\frac{2 E}{m_{e}}\right)^{1 / 2} .
$$

In addition to the photoelectron lifetime broadening, the corehole relaxation also contributes a broadening $\Gamma_{\text {hole }}$. Here we adapt an adiabatic approximation, and so consider only an energy-independent value for the relaxation lifetime $\tau_{\text {hole }}=$ $1 / \Gamma_{\text {hole }}$. As these processes both possess Lorentzian line widths, we can sum them directly to obtain the energydependent Lorentzian broadening of the spectrum,

$$
\Gamma(E)=\Gamma_{\lambda}(E)+\Gamma_{\text {hole }} .
$$

Values for the hole relaxation broadening $\Gamma_{\text {hole }}$ may be provided by the user with the Gamma_hole keyword, but are otherwise included by default from the tabulations of Scofield and Kostroun et al. for $Z=21-50$ (Scofield, 1969; Kostroun et al., 1971), and from Bambynek et al. for $Z=51-100$ (Bambynek et al., 1972). Errors in XAFS spectra associated with the core-hole lifetime are typically observable only for very low photoelectron energies, below $10 \mathrm{eV}$. Note that, especially when simulating high-resolution experiments in fluorescence mode (HERFD, RIXS), the user can optionally reduce the $\Gamma_{\text {hole }}$ value.

With the oscillatory component of the spectrum determined, inclusive of broadening, thermal and static disorder contributions, the atom-like components of the spectrum must be properly quantified in order to isolate the XAFS spectrum for robust analysis. This includes the background absorption both in terms of the $K$-shell contribution, and the contribution from less strongly bound electrons (i.e. the $L$ shells, $M$ shells, valence electrons etc.).

The background $K$-shell contribution to the spectrum, $\mu_{0}(k)$, is estimated via explicit calculations of atomic spectra within the $F D M X$ package. These are performed with small clusters, approximately $1.5 \AA$ in radius, and extremely high grid density in order to attain highly accurate background functions for all possible absorbing elements with $Z=21-92$. These functions are tabulated and output as part of standard $F D M X$ calculations, allowing users to readily extract the oscillatory part of the spectrum for analysis. This method is highly advantageous over traditional spline techniques as it does not introduce artificial low-frequency structures into the spectrum. In some extreme cases, however, care must be taken within a few $\mathrm{eV}$ of the absorption edge due to the potential for solid-state bonding structure to significantly affect the Fermi energy, and thus affect the extracted $\chi(k)$ for $k \lesssim 1.5$.

Contributions to the absorption spectrum from less strongly bound electrons are incorporated using atomic form factor tabulations (Chantler, 1995, 2000). These tabulations are calculated via the FFAST package, which uses the multiconfigurational Dirac-Hartree-Fock technique for the selfconsistent evaluation of relativistic atomic wavefunctions, and a local density approximation (LDA) for the treatment of the Coulomb and exchange-correlation potentials. The photoelectron absorption from FFAST is typically accurate to well within $1 \%$ for energies that are not close to an absorption edge, which is almost always the case for the background contribution from other electrons. Contributions from photon scattering are not included in $F D M X$, so that the output is strictly the photo-absorption, rather than total attenuation.

A final contribution of background absorption is sometimes necessary in order to directly compare theoretical results with experiment spectra on an absolute scale. This is due to the edge-jump discrepancy, sometimes called the triangle effect (Tantau et al., 2015), that is currently common to all photoabsorption computations (Chantler \& Bourke, 2014a). By default this contribution is not included in FDMX, but the user may add an additional background absorption using the Expntl or Victoreen keywords. The contribution will then be added following

$$
\mu(E) \rightarrow \mu(E)[1+\eta(E)]
$$

where $\mu(E)$ is the total photo-absorption coefficient and $\eta(E)$ is a smooth function of the form $\eta(E)=A \exp \left(E-E_{0} / B\right)$ if Expntl is used (Chantler \& Bourke, 2010), or $\eta(E)=A\left(E_{0}^{3} / E^{3}\right)$ $+B\left(E_{0}^{4} / E^{4}\right)$ if Victoreen is used (Victoreen, 1949). In both cases, $E_{0}$ is the absolute edge energy and $A$ and $B$ are parameters.

These functions allow a smooth scaling of the background absorption without artificial oscillatory structures as with a cubic spline, and tend to produce quite good results even for materials where the offset is large (see, for example, Fig. 1). When an exponential function is used, $A$ controls the edge jump scaling and $B$ controls the rate of convergence to the atomic result. For a Victoreen function, $A+B$ gives the scaling and the ratio $A / B$ gives the rate of convergence. 


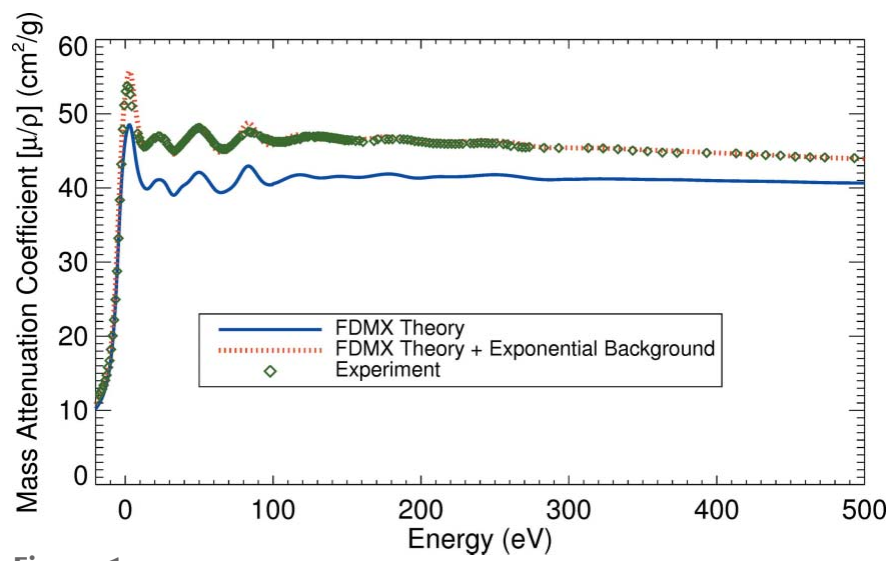

Figure 1

XAFS of elemental Sn, calculated using the $F D M X$ package with default physical parameters, and computational parameters given by Table 1 (solid blue line). Also shown is the same spectrum with an added exponential background function (dotted red line), and high absolute accuracy measured data (de Jonge et al., 2007) (green diamonds).

Typically for a Victoreen function, a negative value is required for $A$.

\section{Computational convergence}

One of the key limitations of the previous implementation of the FDM for the calculation of final-state wavefunctions was the inability to sufficiently sample the potential at high energies within a feasible computational framework. Essentially, the grid density required for convergence became increasingly large at high energies, leading inevitably to either unstable results or impractical calculations (Bourke et al., 2007).

$F D M X$ addresses this issue in a number of ways. Firstly, the grid density now defaults to a variable parameter that increases step-wise as the energy is increased, in order to ensure a proper representation of the electronic potential, and acceptable convergence of the final-state wavefunction. Typically, this can be interpreted as a representation that results in errors in the calculated absorption cross section of $0.1 \%$ or less prior to the introduction of broadening contributions. The grid density, and the rate at which it changes, is adjustable using the adimp keyword in the event that satisfactory convergence is not achieved for a particular molecule. Secondly, the radius of the computational cluster is also changed with increasing energy, in order to ensure that the number of grid points considered remains practical.

The reduction of the cluster size, which may be controlled with the Radius keyword, may result in the loss of outer coordination shells from the calculation at sufficiently high photoelectron energies. In rare cases this may result in the loss of oscillatory structure, and so the user must be conscious of this potential drawback. However, in the vast majority of cases, the oscillatory structure will remain unchanged due to the broadening contribution from the electron IMFP, which will increasingly filter contributions from the more distant neighbouring atoms (Bourke et al., 2007). Therefore it is typically safe, and substantially more efficient, to use default values to define the form of the cluster.
Table 1

Cluster parameters for calculating XAFS of different materials.

The parameters used for $\mathrm{FeO}_{6}$ are the default within FDMX.

\begin{tabular}{|c|c|c|c|c|c|c|}
\hline \multirow{2}{*}{$\begin{array}{l}\text { Energy } \\
\text { range }(\mathrm{eV})\end{array}$} & \multicolumn{3}{|c|}{ Cluster radius $(\AA)$} & \multicolumn{3}{|c|}{ Grid spacing $h(\AA)$} \\
\hline & $\mathrm{Sn}$ & $\mathrm{TiO}_{2}$ & $\mathrm{FeO}_{6}$ & Sn & $\mathrm{TiO}_{2}$ & $\mathrm{FeO}_{6}$ \\
\hline Up to 100 & 8.3 & 8.3 & 8.3 & 0.24 & 0.24 & 0.24 \\
\hline $100-200$ & 6.0 & 6.0 & 7.0 & 0.18 & 0.18 & 0.20 \\
\hline $200-250$ & 6.0 & 4.0 & 7.0 & 0.18 & 0.18 & 0.20 \\
\hline $250-300$ & 4.0 & 4.0 & 5.0 & 0.14 & 0.14 & 0.16 \\
\hline 300-400 & 4.0 & 4.0 & 5.0 & 0.12 & 0.12 & 0.16 \\
\hline $400-500$ & 4.0 & 4.0 & 5.0 & 0.12 & 0.10 & 0.12 \\
\hline
\end{tabular}

In addition to these measures, the most recent implementations of the FDM, including FDMX, feature improved functionality through the use of more efficient data management (Glover et al., 2007) and the employment of the MUMPS Fortran libraries for the manipulation of large data arrays (Amestoy et al., 2001). The MUMPS-related features have been recently implemented for FDM calculations by Guda, Soldatov et al., and enable calculations of absorption spectra up to 30 times faster, and with substantially less memory use, than was previously possible (Guda et al., 2015; Amestoy et al., 2006). These improvements, coupled with the dynamic cluster parameters, allow routine determinations of extended XAFS spectra up to the smooth atom-like region within the space of an hour for simple materials with high symmetry, or a few hours for arbitrary molecular structures.

\section{Example spectra}

To demonstrate the use of the new package, we present example spectra for elemental tin, the mineral rutile $\left(\mathrm{TiO}_{2}\right)$ and the octahedron $\mathrm{FeO}_{6}$. In all cases, default options were used for physical parameters including electron IMFP, thermal effects and background absorption. Computational parameters were varied in some cases in order to ensure convergence, and are given in Table 1. For $\mathrm{TiO}_{2}$ and $\mathrm{FeO}_{6}$, the results are compared with those using the multiple-scattering technique, implemented within the same package and also within FDMNES.

\subsection{Metallic tin, Sn}

We firstly consider elemental tin, the attenuation spectrum for which is shown in Fig. 1. It is compared here with high absolute accuracy experimental data using the standard $\beta$-tin allotrope (de Jonge et al., 2007).

The resulting XANES and XAFS spectra are in very strong agreement with the experimental data, as has previously been shown with prototype $F D M X$ calculations for solid metals (Chantler \& Bourke, 2014a; Tantau et al., 2015). The only significant difference appears in the height of the peak at $83 \mathrm{eV}$, most likely due to an overestimate of the electron IMFP in the default data (Bourke \& Chantler, 2012). A full computation of the IMFP using the self-consistent model of equation (13) provides more accurate results, and is an optional (and unique) feature of $F D M X$. This result using the 
FDM is similar to that using a full multiple-scattering (FMS) approach within the same package (including the additional developments of IMFP, thermal broadening and hole-widths as discussed above), as approximations in the electronic potential are less critical for calculations involving elemental solids.

The dotted red curve in Fig. 1 shows the calculated spectrum with an added exponential scaling using the parameters $A=0.18$ and $B=780 \mathrm{eV}$. A similar background is obtainable for elemental Sn using a Victoreen function with parameters $A=-4.95$ and $B=5.13$. Direct comparison with experimental spectra, and subsequent quantitative analysis, is therefore possible using a simple background function that does not oscillate, even over an extended energy range. This is in contrast with the common cubic spline functions usually used in alternative packages. With this background, we find that the $\chi_{r}^{2}$ discrepancy from experiment is 63.5 over the energy range spanning $-10 \mathrm{eV}$ to $500 \mathrm{eV}$ relative to the edge. This is an excellent result given the high absolute accuracy of the experimental spectrum (typically of order $0.1 \%$ ), lack of optimization of physical parameters, and range of energies that include the edge, XANES and EXAFS regions. The average discrepancy between theory and experiment over this range is $0.77 \%$.

\subsection{Rutile, $\mathrm{TiO}_{2}$}

Fig. 2 shows the calculated spectra for the mineral rutile, $\mathrm{TiO}_{2}$. In this example we make a direct comparison between the result using the full-potential FDM calculation and an equivalent FMS calculation also performed within the FDMX package. As with Sn, default settings are used for all physical parameters for both the FDM and FMS models, while the computational parameters are varied in accordance with Table 1. For the FMS case, grid density parameters are not applicable as a muffin-tin approximation is used, corresponding to a constant potential in the interstitial region.

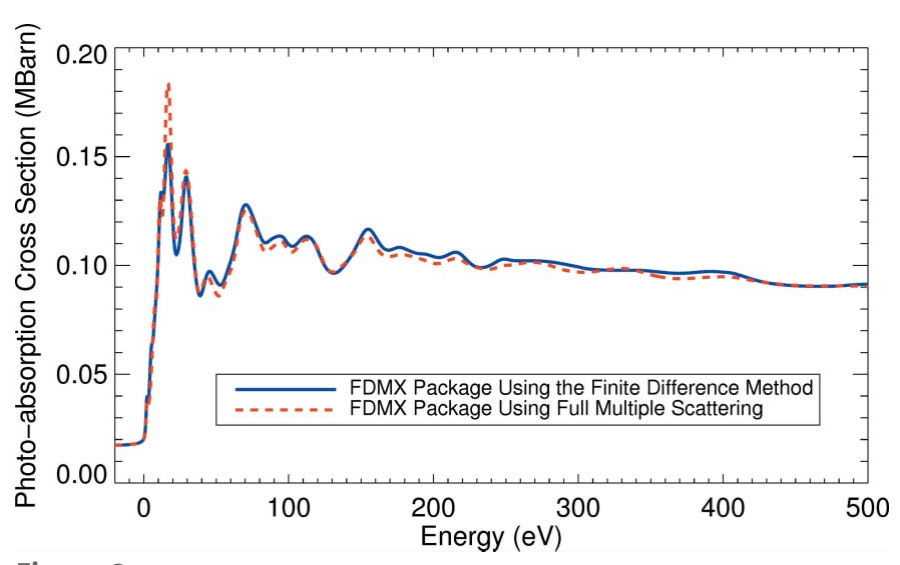

Figure 2

XAFS of rutile $\mathrm{TiO}_{2}$, calculated using the FDMX package with a fullpotential FDM representation (solid blue line), and an equivalent full multiple scattering (FMS) calculation (dashed red line). Both spectra utilize the same physical models in FDMX for thermal motion, lifetime broadening and background processes, meaning that all differences between the spectra result directly from approximations of the FMS approach in the electronic potential.
The spectra resulting from the two models are not convergent for this material even for the majority of the XAFS region, with acceptable agreement only apparent after $400 \mathrm{eV}$, if at all. As the calculations were performed with identical systems within the same package and using the same parameters, all of the observed differences are necessarily due to the approximated form of the potential in the FMS calculation. This FMS potential follows a muffin-tin form, requiring a constant value in the interstitial region, leading to discontinuities in the gradient of the potential and, as this is a compound, in its value at the interface between the regions of the calculation. Strictly speaking, the FDM potential is also approximated due to the finite density of grid points; however, care has been taken to ensure convergence has been reached. For $\mathrm{TiO}_{2}$, this meant that the grid spacing $h$ needed to be reduced below its default value. The clear conclusion is that the $F D M X$ package and approach has advantage in near-edge and central XAFS regions in avoiding mathematical artefacts due to flat interstitial potentials and discontinuities.

This result is highly significant in terms of the choice of model for XAFS calculations with compound materials, especially those with molecular or cluster sites with significant variations of atomic number. The mean variation between the two spectra is $2.0 \%$ between $-10 \mathrm{eV}$ and $500 \mathrm{eV}$. This is significantly higher than the absolute accuracies now obtainable even for complex molecules using precision XAFS techniques (Chantler et al., 2012), and is, for example, almost three times greater than the absolute discrepancy seen in the previous section between theory and experiment for Sn.

In the XANES region, the differences between models are particularly strong, as is expected due to the strong impact of discontinuities in the shape and value of the electronic potential on the reflection of the low-energy electron wavefunction. Indeed, for energies below $60 \mathrm{eV}$, the difference between FDM and FMS spectra is $4.6 \%$. These discontinuities can therefore lead to incorrect conclusions regarding the broadening mechanisms and long-range bonding environment in the studied material.

It is especially notable that the muffin-tin potential can impact XAFS structures in both the XANES and EXAFS regions of the spectrum. Although the impact is strongly material-dependent, it is critical for quantitative XAFS analysis that such errors be minimized, and therefore a fullpotential modelling is strongly advised.

\section{3. $\mathrm{FeO}_{6}$ octahedra}

An octahedral structure $\mathrm{FeO}_{6}$ is modelled in Fig. 3, where we see that implementations of the FDM and FMS models within FDMX can both be useful for high energies. Over the energy range plotted, the difference between the two theoretical models is only $0.86 \%$, which is partly aided by the relatively weak XAFS signal common to disordered structures without long-range order or highly degenerate scattering paths. Nevertheless, key differences between the predicted peaks between the two models are observed, which may prove 


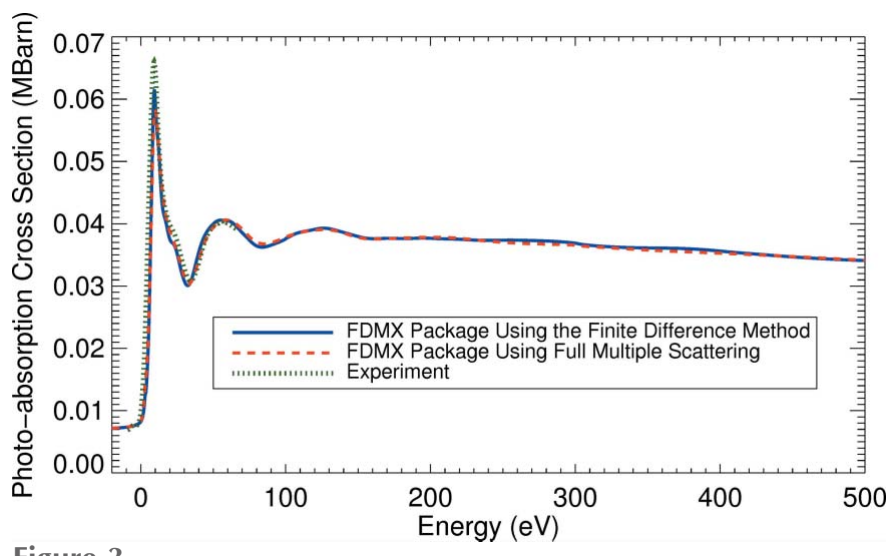

Figure 3

XAFS spectra of the $\mathrm{FeO}_{6}$ octahedron, calculated using the FDMX package using a full-potential FDM representation (solid blue line) and a muffin-tin FMS representation (dashed red line). Also shown for comparison are experimental results in the XANES region (dotted green line).

significant for detailed analysis with highly accurate experimental data.

Most particularly, however, we are here interested in comparing the performance of the two models with experimental measurements in the XANES region. We therefore provide a closer view of the $\mathrm{FeO}_{6}$ spectra in Fig. 4. Here we observe a small offset in the edge-jump, and an offset in the energy of the edge itself. While the former issue may be similar in nature to the effect seen in Sn, the latter issue may be related to small errors in the Fermi energy in the model predictions, approximations from the Hedin-Lundqvist exchange-correlation potential, or properties of the lowenergy band structure of the material that cannot be interpreted within an atomistic model.

The forms of the spectra themselves are an important demonstration of the value of the use of a full-potential model, which in this case is not apparent from a direct comparison of discrepancy values. Up to $70 \mathrm{eV}$, the FDM result deviates from experiment by an average of $6.9 \%$, while the FMS deviates by

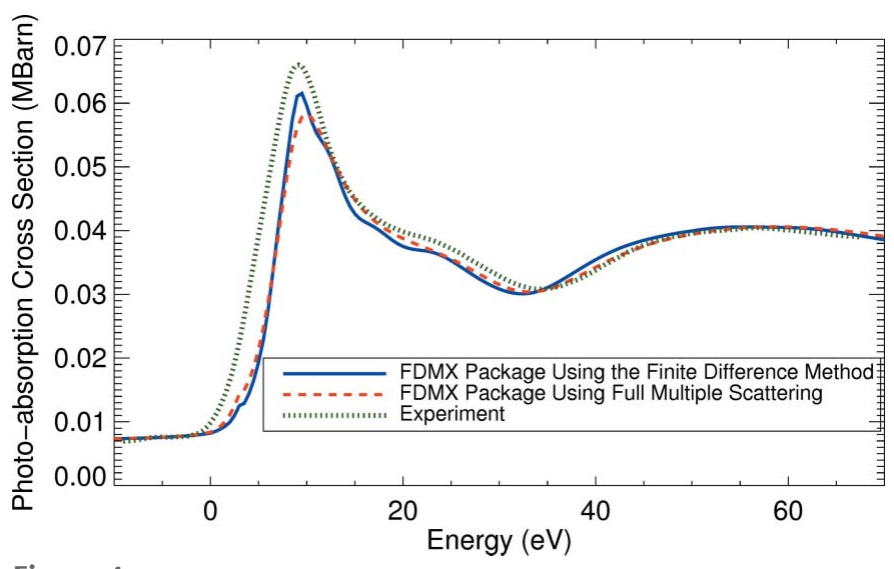

Figure 4

XANES of the $\mathrm{FeO}_{6}$ octahedron, calculated using the $F D M X$ package using a full-potential FDM representation (solid blue line) and a muffintin FMS representation (dashed red line), compared with experimental data (dotted green line). only $5.6 \%$. The theories deviate from one another in this range by an average of $2.0 \%$. In addition to the effects already mentioned, some of the discrepancy with experiment is also due to the model itself, which in this case uses an explicit $\mathrm{FeO}_{6}$ octahedron, while the experimental data were a more complex structure, including a possible second oxygen shell and effects of the hydrogen atoms, with a formal charge of $\mathrm{Fe}^{2+}$ and recorded at the FAME beamline, ESRF (Testamale et al., 2009). Hence we expect to see chemical shifts, small amplitude changes and changes of structure associated with the nonnearest neighbours. In other words, this example is expected to require structural development before achieving perfect agreement with experimental data.

The FDM theory does, however, produce extra structures that are not predicted by the FMS theory. In particular, the FDM theory is able to replicate the characteristic peak at around $25 \mathrm{eV}$ from the absorption edge seen in the experimental result. A peak of this relative magnitude and at this energy is unlikely to be introduced into an FMS calculation by any of the effects discussed, and is therefore an important indicator of the impact of full-potential modelling.

Sometimes such features can be obscured or distorted by approximations in the broadening mechanisms, and most particularly the photoelectron IMFP. An overestimate of the IMFP can lead to extraneous structures that in practice are broadened out in the experimental measurements. This can be expected for molecular structures that do not have a well defined optical ELF (Bourke \& Chantler, 2012), and is most significant in the near-edge region (Bourke \& Chantler, 2010b).

Similar difficulties exist within FMS theories, but may be overlooked as in this case such a model does not predict the small near-edge peaks in the first place. Broadening mechanisms may smooth out artificial structures from theoretical XANES and XAFS spectra, but cannot introduce the scattering paths required to reproduce observed experimental spectra. For this reason the full-potential modelling of FDMX is critically important for the interpretation of near-edge structures, in addition to producing strong agreement with experiment in the XAFS regime. More work is surely needed in the development of these ideas further.

\section{Conclusions}

The FDMX package is the first full-potential package capable of calculating robust and accurate XAFS spectra across all applicable energies within a single self-consistent computation. Over the course of development of the package, this capacity has been shown to enable new extractions of fundamental physical properties from experimental XAFS spectra (Bourke \& Chantler, 2010b; Tantau et al., 2015), which in turn have led to new insights in fundamental theory (Chantler \& Bourke, 2014b; Bourke \& Chantler, 2015).

Full-potential modelling has long been considered necessary for the interpretation of XANES structures, particularly for compounds and molecular samples. This work demonstrates that the advantages of the FDMX approach, while 
strongly material-dependent, can also be significant for energies at least several hundreds of $\mathrm{eV}$ above the absorption edge.

$F D M X$ is currently available by free download from the CNRS website (http://neel.cnrs.fr/spip.php?rubrique1007 \&; lang=en) and from The University of Melbourne, School of Physics X-ray Optics and Synchrotron Science Group (http: //www.ph.unimelb.edu.au/ chantler/opticshome/softwarepack agedownloads.html\#FDMX).

\section{Acknowledgements}

The authors acknowledge the contributions of their collaborators N. A. Rae, J. L. Glover, L. J. Tantau and M. T. Islam in testing and assisting the development of ideas that lead to this work.

\section{References}

Ambrosch-Draxl, C. \& Sofo, J. O. (2006). Comput. Phys. Commun. 175, $1-14$.

Amestoy, P. R., Duff, I. S., Koster, J. \& LExcellent, J.-Y. (2001). SIAM J. Matrix Anal. Appl. 23, 15.

Amestoy, P. R., Guermouche, A., L'Excellent, J. \& Pralet, S. (2006). Parallel Comput. 32, 136-156.

Bambynek, W., Crasemann, B., Fink, R. W., Freund, H.-U., Mark, H., Swift, C. D., Price, R. E. \& Rao, P. V. (1972). Rev. Mod. Phys. 44, 716-813.

Beni, G. \& Platzman, P. M. (1976). Phys. Rev. B, 14, 1514-1518.

Blaha, P., Schwarz, K., Madsen, G. K. H., Kvasnicka, D. \& Luitz, J. (2001). WIEN2k, An Augmented Plane Wave + Local Orbitals Program for Calculating Crystal Properties. Vienna University of Technology, Austria.

Bourke, J. D. \& Chantler, C. T. (2010a). Nucl. Instrum. Methods Phys. A, 619, 33-36.

Bourke, J. D. \& Chantler, C. T. (2010b). Phys. Rev. Lett. 104, 206601206604.

Bourke, J. D. \& Chantler, C. T. (2012). J. Phys. Chem. A, 116, $3202-$ 3205.

Bourke, J. D. \& Chantler, C. T. (2015). J. Phys. Chem. Lett. 6, 314-319.

Bourke, J. D., Chantler, C. T. \& Witte, C. (2007). Phys. Lett. A, 360, 702-706.

Chantler, C. T. (1995). J. Phys. Chem. Ref. Data, 24, 71.

Chantler, C. T. (2000). J. Phys. Chem. Ref. Data, 29, 597.

Chantler, C. T. \& Bourke, J. D. (2010). J. Phys. Chem. Lett. 1, 24222427.

Chantler, C. T. \& Bourke, J. D. (2014a). J. Phys. Condens. Matter, 26, 145401.

Chantler, C. T. \& Bourke, J. D. (2014b). Phys. Rev. B, 90, 174306.
Chantler, C. T., Rae, N. A., Islam, M. T., Best, S. P., Yeo, J., Smale, L. F., Hester, J., Mohammadi, N. \& Wang, F. (2012). J. Synchrotron Rad. 19, 145-158.

Da, B., Shinotsuka, H., Yoshikawa, H., Ding, Z. J. \& Tanuma, S. (2014). Phys. Rev. Lett. 113, 063201.

Denton, C. D., Abril, I., Garcia-Molina, R., Moreno-Marín, J. C. \& Heredia-Avalos, S. (2008). Surf. Interface Anal. 40, 1481-1487.

Fornasini, P., a Beccara, S., Dalba, G., Grisenti, R., Sanson, A., Vaccari, M. \& Rocca, F. (2004). Phys. Rev. B, 70, 174301.

Glover, J. L., Chantler, C. T., Soldatov, A. V., Smolentsev, G. \& Feiters, M. C. (2007). AIP Conf. Proc. 882, 625-627.

Greegor, R. B. \& Lytle, F. W. (1979). Phys. Rev. B, 20, 4902-4907.

Guda, S. A., Guda, A. A., Soldatov, M. A., Lomachenko, K. A., Bugaev, A. L., Lamberti, C., Gawelda, W., Bressler, C., Smolentsev, G., Soldatov, A. V. \& Joly, Y. (2015). J. Chem. Theory Comput. 11, 4512.

Hedin, L. \& Lundqvist, B. I. (1971). J. Phys. C, 4, 2064-2083.

Joly, Y. (2001). Phys. Rev. B, 63, 125120-125130.

Joly, Y., Cabaret, D., Renevier, H. \& Natoli, C. R. (1999). Phys. Rev. Lett. 82, 2398-2401.

Jonge, M. D. de, Tran, C. Q., Chantler, C. T., Barnea, Z., Dhal, B. B., Paterson, D., Kanter, E. P., Southworth, S. H., Young, L., Beno, M. A., Linton, J. A. \& Jennings, G. (2007). Phys. Rev. A, 75, 032702.

Kostroun, V. O., Chen, M. H. \& Crasemann, B. (1971). Phys. Rev. A, 3, 533-545.

Mermin, N. D. (1970). Phys. Rev. B, 1, 2362-2363.

Nikjoo, H., Uehara, S. \& Emfietzoglou, D. (2012). Interaction of Radiation with Matter. Boca Raton: CRC Press.

Penn, D. R. (1987). Phys. Rev. B, 35, 482-486.

Powell, C. J. \& Jablonski, A. (2009). Nucl. Instrum. Methods Phys. Res. A, 601, 54-65.

Rehr, J. J., Kas, J. J., Prange, M. P., Sorini, A. P., Takimoto, Y. \& Vila, F. (2009). C. R. Phys. 10, 548-559.

Rosen, A. \& Ellis, D. E. (1975). J. Chem. Phys. 62, 3039.

Scofield, J. H. (1969). Phys. Rev. 179, 9-16.

Sorini, A. P., Kas, J. J., Rehr, J. J., Prange, M. P. \& Levine, Z. H. (2006). Phys. Rev. B, 74, 165111.

Tantau, L. J., Chantler, C. T., Bourke, J. D., Islam, M. T., Payne, A. T., Rae, N. A. \& Tran, C. Q. (2015). J. Phys. Condens. Matter, 27, 266301.

Tanuma, S., Powell, C. J. \& Penn, D. R. (1991). Surf. Interface Anal. 17, 911-926.

Tanuma, S., Powell, C. J. \& Penn, D. R. (2011). Surf. Interface Anal. 43, 689-713.

Testamale, D., Brugger, J., Liu, W., Etschmann, B. \& Hazemann, J.-L. (2009). Chem. Geol. 264, 295-310.

Tung, C. J., Ashley, J. C. \& Ritchie, R. H. (1979). Surf. Sci. 81, 427-439.

Victoreen, J. A. (1949). J. Appl. Phys. 20, 1141.

Vila, F. D., Rehr, J. J., Rossner, H. H. \& Krappe, H. J. (2007). Phys. Rev. B, 76, 014301. 